\title{
ERRATUM
}

G.A. Bush - F.A. Miles

\section{Short-latency compensatory eye movements associated with a brief period of free fall}

Exp Brain Res (1995) 108:337-340

The regression line shown in Fig. $2 \mathrm{~A}$ is incorrect and indicates a latency $2.1 \mathrm{~ms}$ shorter than the value given by the correct regression line. This error does not affect any of the quantitative data reported in the paper. 